\title{
The Measurement and Modeling of a P2P Streaming Video Service*
}

\author{
Peng Gao ${ }^{12}$, Tao $\mathrm{Liu}^{2}$, Yanming $\mathrm{Chen}^{2}$, Xingyao $\mathrm{Wu}^{2}$, \\ Yehia El-khatib ${ }^{3}$, and Christopher Edwards ${ }^{3}$ \\ ${ }^{1}$ Graduate University of Chinese Academy of Sciences, Beijing, China \\ ${ }^{2}$ China Mobile Group Design Institute Co., Ltd, Beijing,China \\ \{gaopeng,liutao1,chenyanming,wuxingyao\}@cmdi.chinamobile.com \\ ${ }^{3}$ Computing Department, Lancaster University, Lancaster LA1 4WA, UK \\ \{yehia,ce\}@comp.lancs.ac.uk
}

\begin{abstract}
Most of the work on grid technology in the video area has been generally restricted to aspects of resource scheduling and replica management. The traffic of such a service has a lot of characteristics in common with that of the traditional video service. However the architecture and user behavior in grid networks are quite different from those of the traditional Internet. Considering the potential of grid networks and video sharing services, measuring and analyzing P2P IPTV traffic are important and fundamental works in the field of grid networks.

This paper investigates the features of PPLive, the most popular streaming service in China and based on P2P technology. Through monitoring and analyzing PPLive traffic streams, the characteristics of P2P streaming services have been studied. The analyses are carried out in respect of bearing protocols, geographical distribution and the selfsimilarity properties of the traffic. A streaming service traffic model has been created and verified with the simulation. The simulation results indicate that the proposed streaming service traffic model complies well with the real IPTV streaming service. It can also function as a step towards studying video-sharing services on grids.
\end{abstract}

Keywords: gird, peer to peer, p2p, video streaming, traffic characteristics, traffic modeling, ns2 simulation

\section{Introduction}

Along with the rapid development of P2P file sharing and IPTV video services, P2P streaming services have become the main multi-user video sharing application on the Internet. The focus of grid technology in the video area is generally on the resource schedule and replica management aspect [1] [2], wherein the service traffic characteristic is still similar to the traditional video service. In depth work has been carried out in the areas of monitoring and modeling video traffic [3]. Therefore, considering the developing trends of grid systems and video sharing, monitoring and analysis of P2P IPTV traffic are interesting and promising topics of research. They are the main focus of this paper.

* This work is partially supported by the European Sixth Framework Program (FP6) under the contract STREP FP6-2006-IST-045256. 
There have been many research efforts in the area of P2P live streaming. Silverston et al. [4] have analyzed and compared the different mechanisms and traffic patterns of four mainstreams P2P video applications. Their work has focused on IPTV system aspects such as peer survival time. Hei et al. [5] have made a comprehensive investigation of aspects such as the P2P live streaming system architecture, user behavior, user distribution and software realization. The work presented in these two papers is similar in part to the work presented in this paper. However, our objectives and outcome are dissimilar from theirs in two ways. First, the aim of traffic monitoring is to obtain the characteristics of service traffic. The traffic is analyzed in order to come up with models that can be used in simulation. Second, some of our traffic analysis results differ from those found in the two aforementioned papers for the PPLive software update [7].

The rest of this paper is organized as follows: section 2 describes the monitoring setup, and section 3 details the results of analyzing the measurement data. In order to verify the accuracy of the analysis, a traffic model is developed and evaluated through simulation in Section 4.

\section{Measurement Setup}

The measurement was carried out in the Intranet of China Mobile Group Design Institute (CMDI) in Beijing. Fig. 1 shows the measurement environment, where the Intranet access bandwidth is $100 \mathrm{Mbps}$ and Internet access bandwidth is $10 \mathrm{Mbps}$.

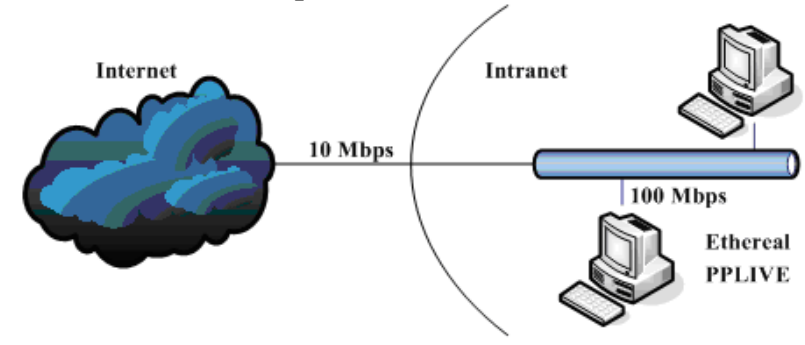

Fig. 1. PPLive Measuring Environment

Monitoring terminals choose a representative popular channel at a peak time to collect the service data. The duration of the data measurement is about 2 hours from the start till the end of the service. The size of the produced data file is $896 \mathrm{MB}$ stored in pcap format. In order to both simplify the data analysis process and to make it sufficiently detailed, the measurement data is divided into several parts, from which two portions of the data (the service beginning and service steady stage) are chosen as the emphases of the analysis. The monitoring terminal runs the PPLive software (version 1.8.18 build 1336). The service uses the default configuration: network type is community broadband, the maximum number of connections per channel is 30 , and the maximum simultaneous network connections is 20. Monitoring terminals use Ethereal version 0.99.0 [8] to collect the traffic data generated by PPLive service. 
Measurement data processing is completed using Gawk [9] and the plots are drawn using gnulpot [10]. The mathematical analysis tool Easyfit [11] is used.

\section{Measurement Data Analysis}

This section presents the results of analyzing different aspects of the measurement data. Since the amount of captured data is relatively large, five identical time sections are selected, where each section is ten minutes long and includes service establishment, middle and end periods. The five time sections are 0-600s, 1800-2400s, $3600-4200$ s, 5400-6000s and 6000-7200s. Section 3.1 uses the data from the 36004200 s time section for analysis, describing the bearing protocols and the sizes of the packets used to transfer both data and control signals. Section 3.2 presents the geographical distribution characteristics of the peers during all the five time sections. Section 3.3 introduces the characteristics of the upload and download traffic based on the first two measurement time sections. The data from the 5400-6000s time section is used in Section 3.4 to investigate self-similarity properties of the traffic.

\subsection{General Measurement}

In order to study network transport protocols that deliver the video streams, a period with relatively steady traffic has to first be considered. The 3600-4200s time section was selected. Fig. 2 shows the size distribution of TCP packets (green dots) and UDP datagrams (red dots) during the mentioned time section. The total number of TCP packets observed during this time section is 462 which is less than $0.46 \%$ of the total number of packets (101620). Further, the size of TCP packets is generally small. On the other hand, UDP datagrams clearly seem to be one of two types. One type is datagrams that are bigger than 600 bytes, most being 1145 bytes long. The other type is datagrams of 100 to 200 bytes. The former type composes $96 \%$ to all the traffic while the latter type composes only $4 \%$. We conclude from this figure that PPLive uses UDP as the main bearing protocol for video content, with packet majorly being 1145 bytes long. TCP is used to transmit some control frames and a few video frames.

\subsection{Peer IP Address Distribution}

Since data collection is on the Chinese financial information channel, the viewers mainly come from China's inland areas. According to the IP address distribution information from China Internet Network Information Center [12], the information of different viewers' location of upload and download can be determined by their IP address. Fig. 3 shows the information of the geographical distribution of IP address. The analysis of the distribution of peers is based on the data in the five time sections which are $0 \sim 600$ seconds, $1800 \sim 2400$ seconds, $3600 \sim 4200$ seconds, $5400 \sim 6000$ seconds and $6000 \sim 7200$ seconds respectively. 


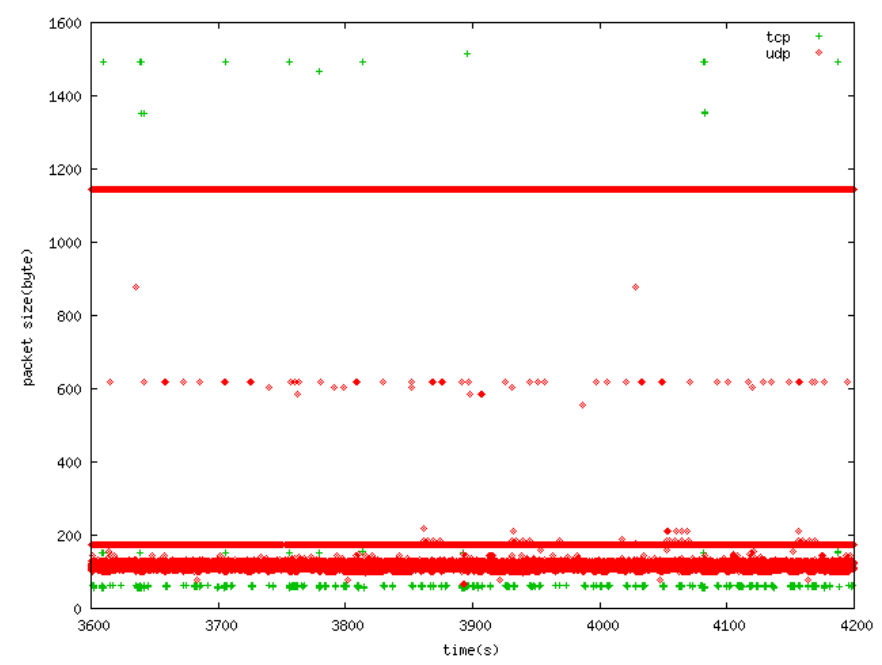

Fig. 2. Packet Size Distribution per Bearing Protocol

The download and upload traffic are processed respectively. The focus of analysis of the peer distribution is on the relationship between the amount of service traffic and corresponding source-area or destination-area. In each time section, the peer IP addresses are sorted according to the number of video packets. Then, the amount of traffic based on the peer IP addresses is converged based on the geographic area of IP address. Fig. 3 shows the traffic ratio from or to different geographic areas in five time sections. The red block represents the first contributing area or beneficial area, the yellow represents the second one, the black represents the third one and the blue represents the set of other areas.

In the download traffic, the contributors are from about 20 provinces. Fig. 3-a shows that more than $50 \%$ traffic are from the first contributing area. In upload traffic, the beneficiaries are from about 25 provinces.
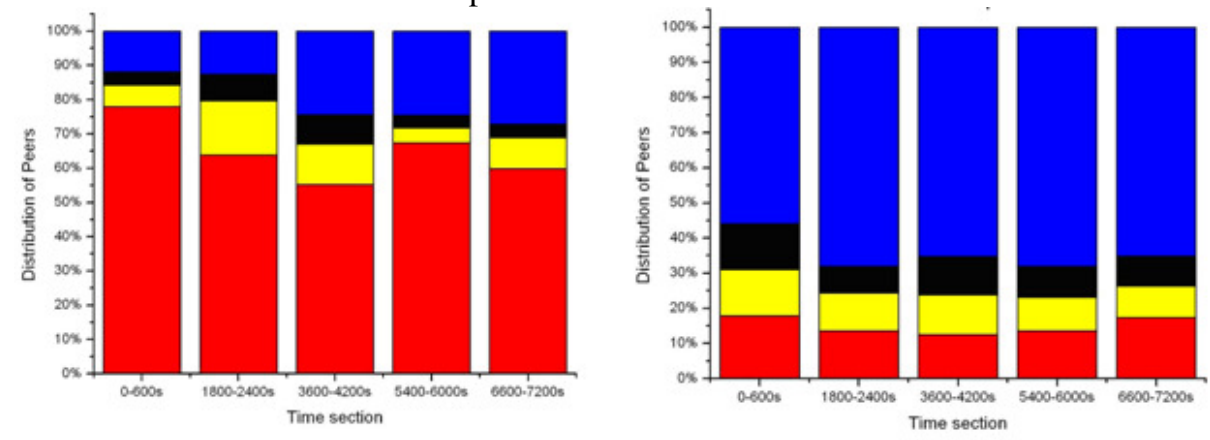

Fig. 3. Peer Distribution for (a) Download and (b) Upload Traffic

Compared with the download traffic, the upload traffic is evenly distributed to different beneficial areas, as shown in Fig.3-b. It is interesting that the download 
traffic is mainly from the network operated by China Necom (CNC) while the upload traffic being mainly to the network operated by China Telecom (CT). The ratio of the traffic is shown in Table1.

Table 1 - Traffic Distribution of the Different Time Sections

\begin{tabular}{|l|c|c|c|c|c|}
\hline Operator & $\mathbf{0 - 6 0 0 s}$ & $\begin{array}{l}\mathbf{1 8 0 0 -} \\
\mathbf{2 4 0 0 s}\end{array}$ & $\begin{array}{l}\mathbf{3 6 0 0 -} \\
\mathbf{4 2 0 0 s}\end{array}$ & $\begin{array}{l}\mathbf{5 4 0 0 -} \\
\mathbf{6 0 0 0 s}\end{array}$ & $\begin{array}{l}\mathbf{6 6 0 0}- \\
\mathbf{7 2 0 0 s}\end{array}$ \\
\hline $\begin{array}{l}\text { CNC } \\
(\text { Download })\end{array}$ & $86.9 \%$ & $86.2 \%$ & $68.78 \%$ & $84.3 \%$ & $80.7 \%$ \\
\hline $\begin{array}{l}\text { CT } \\
\text { (Upload) }\end{array}$ & $70 \%$ & $80.7 \%$ & $56.3 \%$ & $69.2 \%$ & $85.1 \%$ \\
\hline
\end{tabular}

\subsection{Traffic Characteristics}

The upload and download throughput during the first two time sections are shown in Fig.4. Diagrams at different time sections correspond to different time and throughput. Fig. 4 shows the green download traffic presents relatively steady flow rate during the whole data monitoring period, the average is about $50 \mathrm{~KB} / \mathrm{s}$ and there is no dramatic fluctuation from the beginning to the end. The red upload traffic can be divided into two periods, which are service beginning period and the service steady period. During the service beginning period, there is an obvious increasing process. The time point is around the 10th minute and before the 10th minute, there are mainly the detect packets and there is no video packet transmission. During the service steady period, data packets are transmitted according to the link condition of the user.

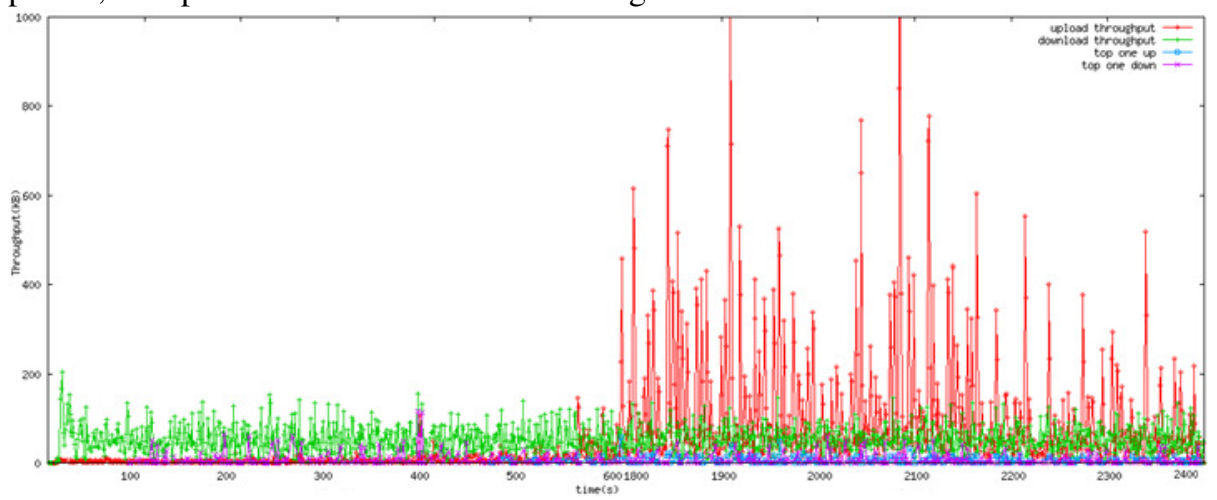

Fig. 4. Upload and Download Traffic Performance with Each Top One Peer Traffic

Fig.4 also shows the top peer traffic both in download and upload, expressed in blue and purple separately, which represent the policy of download and upload. It can be noticed that the output traffic to the top one beneficiary almost equals the contribution from the top one contributor in the download traffic. The statistic granularity is also one second. The average of the rate of the upload and download top one peer is about $12 \mathrm{KByte} / \mathrm{s}$. 
The relationship between the node and the throughput is analyzed with reference to the data between 1800 s and 2400 s and here only the upload traffic is analyzed. The beneficiaries are sequenced according to their received traffic.

Compared with the download traffic, the number of the accumulated nodes of the upload traffic is larger. Therefore, the upload traffic obtained by different nodes is comparatively less. The top one node which obtains the most upload traffic only obtains $5 \%$ of the overall upload traffic. The upload traffic output decreases obviously after the 60th peer and after the 90th peer, there is basically only the output of control packets. During the overall monitoring period, only 4\% of peers (top 100 peers) receive $96 \%$ of data traffic (the determination of the number of nodes is based on the IP addresses) and the $96 \%$ upload traffic is composed of 1145-byte video packet and the remaining part is composed of 100-200Byte detect packets.

\subsection{Self-Similarity Analysis}

The analysis of the self-similar and long range dependence is achieved by the Selfis tool [13] from Thomas Karagiannis et al. The data in the 5400-6000s time section is used as the analysis basis of download and upload traffic. Fig.5 shows both the download and upload traffic analysis results of R/S estimation and autocorrelation function respectively. The R/S linear fitting function is $0.1723 \mathrm{x}+0.01451$, and the self-similar Hurst value is 0.1732. According to the increasing lags, the autocorrelation function has no notable decline and its characteristic of non-summable is not clear. For the upload traffic, its R/S linear fitting function is $0.6216 x+0.0349$, and the corresponding Hurst value is 0.6216 . The non-summable characteristic of autocorrelation function is also unclear, but since the Hurst value is more than 0.5 , it can be regarded that the ACF of the upload traffic is non-summable $(\mathrm{H}>0.5)$, i.e. the upload traffic is long range dependent.

According to the analysis of several time sections of upload and download traffic, the Hurst value of download traffic fluctuates from the 0.2 to 0.3 , and the Hurst value of upload traffic fluctuates from 0.6 to 0.7 . If the data analysis is in a long time scale, i.e. more than 50min, the Hurst values of download and upload traffic will obviously increase which are at 0.35 and 0.75 respectively, and the ACF has no clear decline either.

\subsection{Traffic Statistical Fitting}

From the analysis of the above sections, we draw two conclusions about modeling the traffic of PPLive. Firstly, traffic modeling of the PPLive service should be based on the upload traffic. Secondly, for simplicity, the upload traffic should be modeled as a whole, although actually being composed of traffic to different beneficiaries.

Detecting video packets groups interval time, the fitting PDF and its corresponding parameters based on the K-S test sequencing are considered, as shown in Table 2 below. log-normal PDF is chosen as the video frame transmitting function of traffic modeling. 

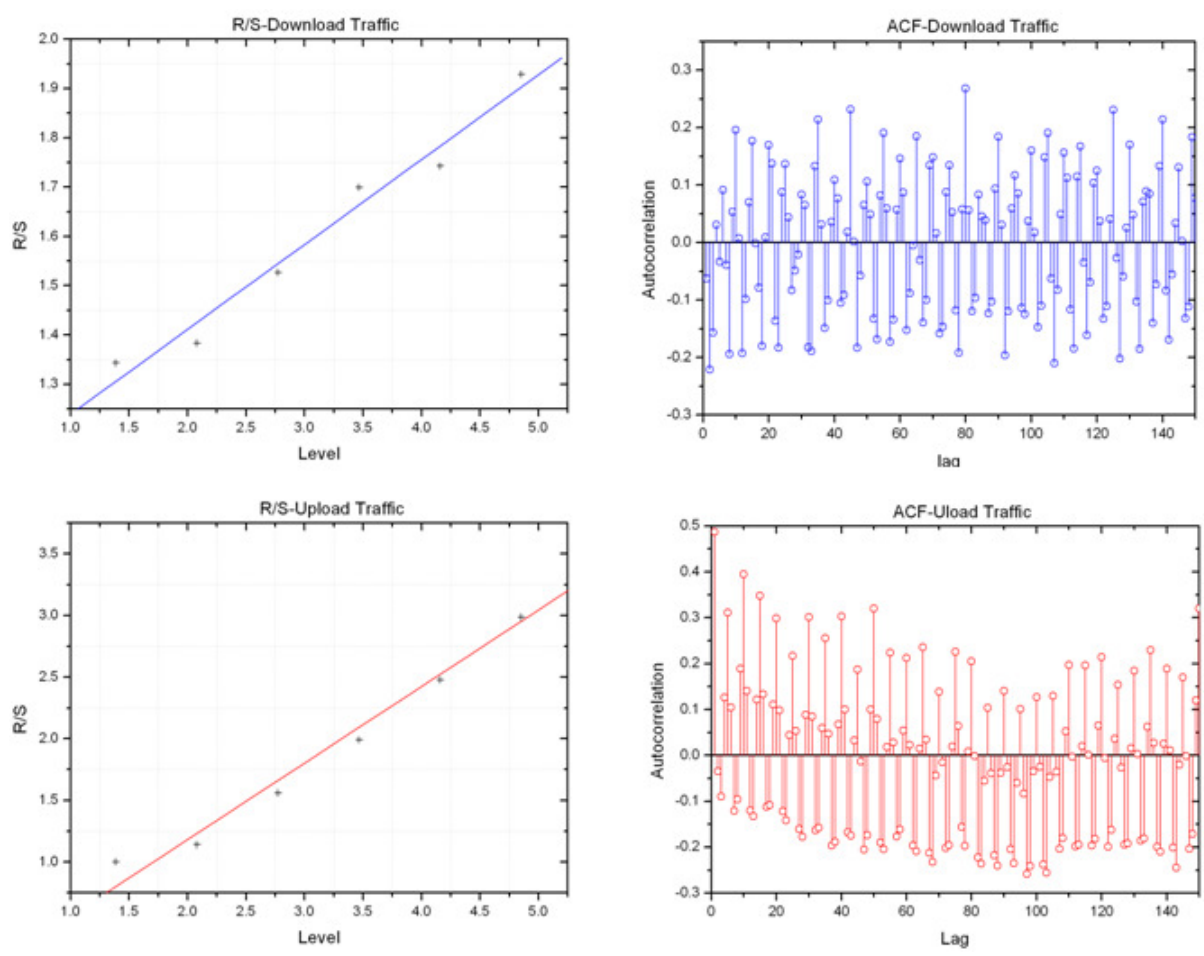

Fig. 5. R/S and ACF of Download and Upload Traffic

Table 2. PDF Fitting.

\begin{tabular}{|c|c|c|}
\hline Distribution & K-S Test & Parameter \\
\hline LogNormal & 0.03177 & $\sigma=1.5514 \mu=-3.7143$ \\
\hline LogNormal (3p) & 0.03385 & $\begin{array}{c}\sigma=1.7522 \mu=-3.86 \\
\gamma=9.6370 \mathrm{E}-4\end{array}$ \\
\hline Weibull (3P) & 0.03614 & $\begin{array}{c}\alpha=0.61622 \beta=0.04795 \\
\gamma=0.0011\end{array}$ \\
\hline Gamma (3P) & 0.07984 & $\begin{array}{c}\alpha=0.53457 \beta=0.12649 \\
\gamma=0.0011\end{array}$ \\
\hline Weibull & 0.08644 & $\alpha=0.62782 \beta=0.05058$ \\
\hline
\end{tabular}

Fig. 6 shows the Q-Q plot of the log-normal distribution, which indicates the best $\mathrm{K}-\mathrm{S}$ test distribution when analyzing the video packets inter time. It is shown that before 0.4 seconds the log-normal distribution can match the actual data well but deflect gradually thereafter. However, since the inter packets time is all less than $3 \mathrm{~s}$ and almost $92 \%$ of all is less than 0.4 second, this does not influence the traffic model much. 


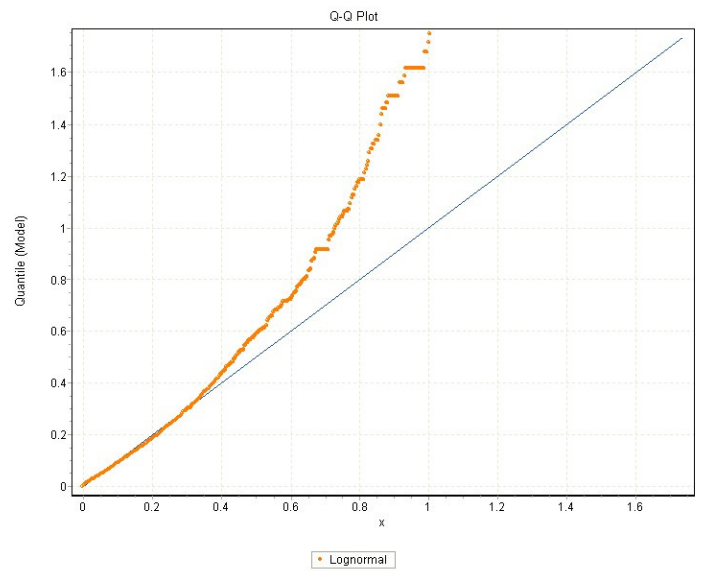

Fig. 6. Statistic Fitting of Video Packet

We then use the same way to analyze the control packets. The fitting PDF of small packet time interval and its corresponding parameters based on the K-S test sequencing are considered. Again, log-normal PDF is chosen as the control packets function of traffic modeling.

\section{$4 \quad$ Modeling Design and Simulation}

As we know, the actual net system is very heterogeneous and complicated. Managing and developing such a large-scale distributed system is very difficult and hardly feasible, so modeling is the best way to simulate its operation. This paper provides an effective method to model IPTV traffic. This method can serve as a reference for similar work in the future. On the other hand, the simulation results can also verify the accuracy of the analysis and modeling methods used.

\subsection{Algorithm}

In this model, an algorithm has been put forward to control the packet generation sequence, as shown in Fig. 7.

First, data initialization is performed.

1. Send a video packet when simulation begins.

2. Compute the next video packet sending time.Put it into a variable NextT.

Next, the time needed to send the next packet is computed. To account for different packet sizes, different parameters are used to calculate inter- video packet time (variable NextT) and the inter-control packet time(array $\left.t \_i\right)$. The values ofe $t \_1$ to $t \_n$ are summed to variable SmallT. As long as the value of SmallT is less than NextT, t_i is used as the inter-packet time for sending small packets. Otherwise, a large packet is sent immediately with an inter-packet time of NextT - (SmallT - t_i). 


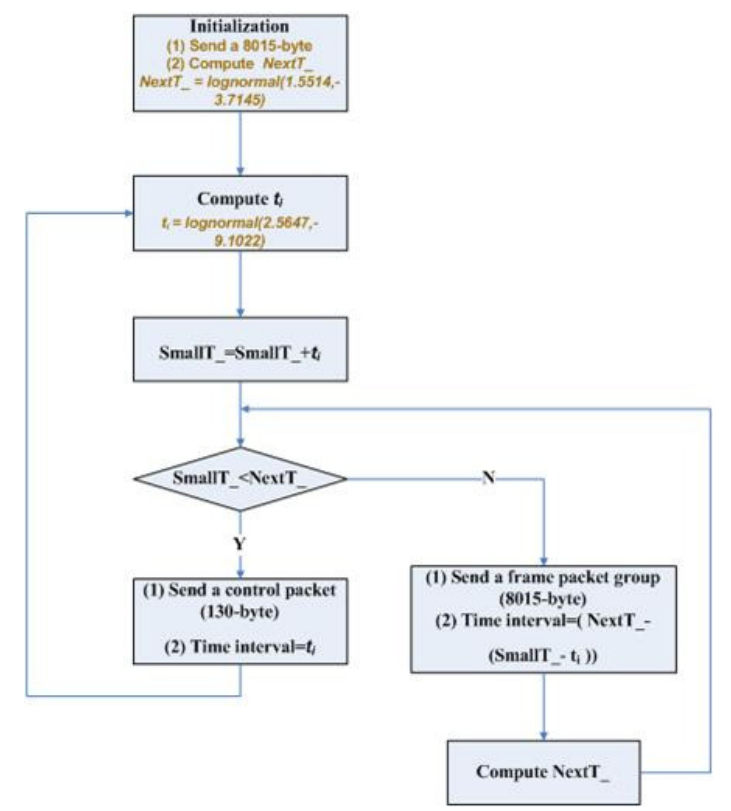

Fig. 7. Packet Generation Sequence Flowchart

\subsection{Simulation and Evaluation}

For the simulation, version 2.29 of the widely-used NS-2[14] is used to implement the model. Script analysis language Awk, mapping tool gnuplot and statistical fitting tool Easyfit are all used here for the simulation data analysis.

The log-normal distribution with the parameters $\alpha$ equals 1.5514 and $\beta$ equals 3.7143 is used to simulate the inter-video packet time interval, as discussed before. Log-normal with parameters $\alpha$ equals to 2.5647 and $\beta$ equals to -9.1022 simulate the inter-packet time for small packets, as explained in Section 4.1, while the size of control packets is 130 and the size of video packets is 8015 .

Fig. 8 shows the Q-Q plot of the characteristics of inter-video packet time from simulation, which match very well during the first 2 seconds and then depart gradually compared with the actual data.

In order to further validate the correctness of this traffic model, ten minutes of simulation traffic data has been collected to draw a throughput plot. This is compared with the data from the actual throughput for 3600-4200s by plotting them on the same figure, as shown in Fig. 9. The red points are real traffic data and the blue points are the simulation data. 


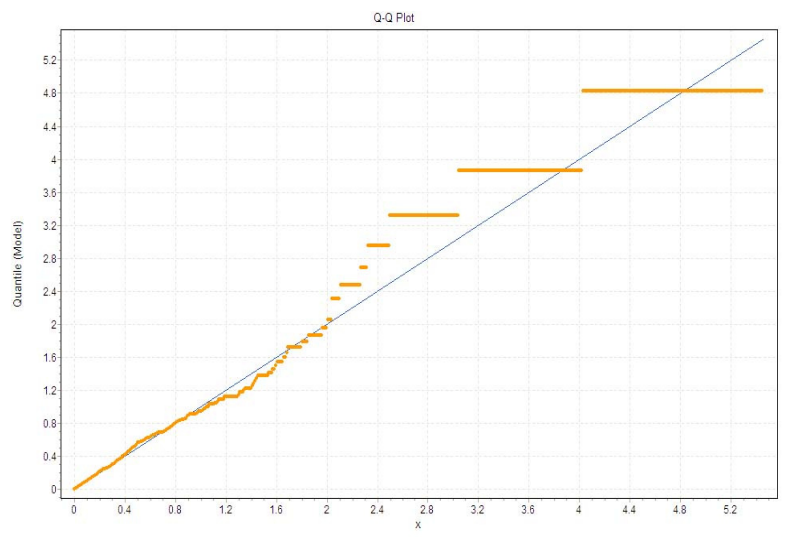

$\cdot \cdot$ Lognormal

Fig. 8. Q-Q Plot of Packets Inter-Arrival Time

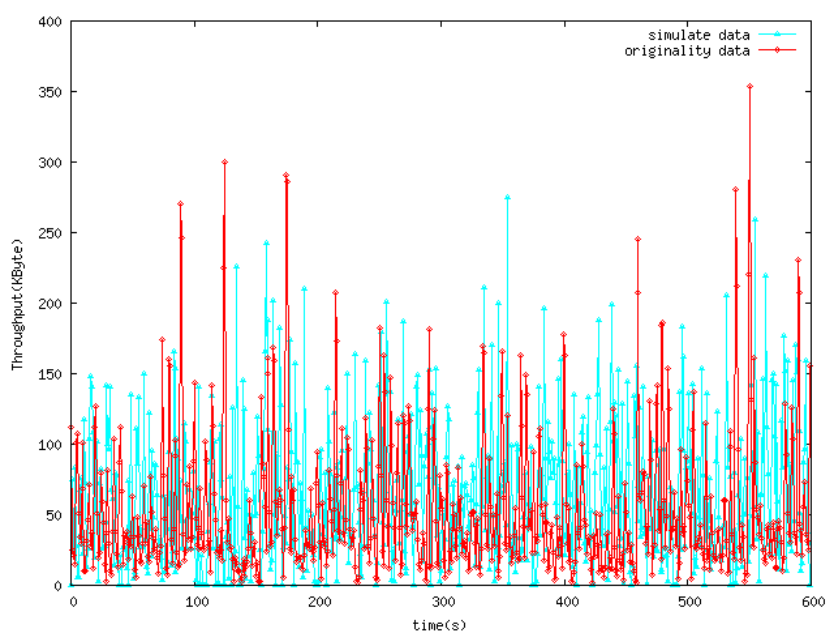

Fig. 9. Comparison between Original and Simulated Traffic

The performance of these two sets of traffic data are compared in Table 4, which indicates that this model can be used to represent traffic generated by IPTV peers on a simulation platform. 
Table 3 - Comparing data

\begin{tabular}{|c|c|c|c|c|}
\hline Data & $\begin{array}{c}\text { Total Traffic } \\
\text { (Kbyte) }\end{array}$ & $\begin{array}{c}\text { Average } \\
\text { Value } \\
\text { (Kbyte/s) }\end{array}$ & $\begin{array}{c}\text { Standard } \\
\text { Deviation }\end{array}$ & $\begin{array}{c}\text { Confidence } \\
\text { Level (95\%) }\end{array}$ \\
\hline Actual & 30907 & 51.59766 & 51.0418 & 4.0958 \\
\hline Simulation & 38197 & 63.76795 & 52.0086 & 4.1734 \\
\hline
\end{tabular}

\section{Conclusion}

This paper investigates features of PPLive, a representative of P2P Video Streaming technology, such as distribution of peer IP addresses, number of peers, and various traffic characteristics including self-similarity. By further analysis, we designed a model for the streaming video traffic generated by PPLive. We verified the correctness of our model using simulations of the video streaming service. The results indicate that the proposed streaming service traffic model complies with the actual IPTV streaming service. This study of IPTV traffic streams can be used in the future as a foundation for characterizing grid video-sharing services.

\section{References}

1. Cuilian L, Yunsheng M, Junchun R. "A Grid-based VOD System and Its Simulation". Journal of Fudan University (Natural Science), Vol.43 No.1, pp. 103-109, Feb 2004.

2. Anfu W. "Program Scheduling and Implementing in a Grid-VOD System". Engineering Journal of Wuhan University, Vol.40 No.4, pp. 149-152, August 2007.

3. Garrett MW, Willinger W. "Analysis, Modeling and Generation of Self-Similar VBR Video Traffic". Proceedings of SIGCOMM '94, September 1994.

4. Silverston T, Fourmaux O. "Measuring P2P IPTV Systems". Proceedings of the $17^{\text {th }}$ ACM International Workshop on Network and Operating Systems Support for Digital Audio \& Video (NOSSDAV'07), Urbana, IL, USA, pp. 83-88, June 2007.

5. Hei X, Liang C, Liang J, Liu Y, Ross KW. "A Measurement Study of a Large-Scale P2P IPTV System". IEEE Transactions on Multimedia, volume 9, issue 8, pp. 1672-1687, October 2007.

6. El-khatib Y, Edwards C (eds.), Damjanovic D, Heiß W, Welzl M, Stiller B, Gonçalves P, Loiseau P, Vicat-Blanc Primet P, Fan L, Wu J, Yang Y, Zhou Y, Hu Y, Li L, Li S, Liu S, Ma X, Yang M, Zhang L, Kun W, Liu Z, Chen Y, Liu T, Zhang C, Zhang L. "Survey of Grid Simulators and a Network-level Analysis of Grid Applications". EC-GIN Deliverable 2.0, University of Innsbruck, April 2008.

7. PPLive http://www.pplive.com/

8. Ethereal: A Network Protocol Analyzer. http://www.ethereal.com/

9. Gawk. http://www.gnu.org/software/gawk/

10. gnuplot. http://www.gnuplot.info/

11. Easyfit: Distribution Fitting Software. http://www.mathwave.com

12. China Internet Network Information Center http://www.cnnic.net.cn/en/index/ index.htm

13. The SELFIS Tool. http://www.cs.ucr.edu/ tkarag/Selfis/Selfis.html

14. NS-2: Simulation tool. http://nsnam.isi.edu/nsnam/ 


\section{Acknowledgment}

First of all, we thank the Europe-China Grid InterNetworking (EC-GIN) project which provided a wonderful platform for in-depth research on Grid networks. We are indebted to the members of the EC-GIN WP2 group for their collaboration and support. Our team members tried their best, and now we can share the success. We also express our gratitude to Michael Mackay for his valuable comments. Finally, we thank all the people whose experience we have benefited from, and those concerned with the improvement of Grids! 